9th A. Friedmann International Seminar and

3rd Casimir Symposium 2015

International Journal of Modern Physics: Conference Series

Vol. 41 (2016) 1660132 (11 pages)

(C) The Author(s)

DOI: $10.1142 /$ S2010194516601320

\title{
Extending the geometric deformation: New black hole solutions
}

\author{
Jorge Ovalle \\ Abdus Salam International Center for Theoretical Physics (ICTP), \\ Strada Costiera 11, Trieste 34014, Italy \\ Dipartimento di Fisica e Astronomia, Alma Mater Università di Bologna, \\ Bologna 40126, Italy \\ Departamento de Física, Universidad Simón Bolívar, Apartado 89000, \\ Caracas 1080 A, Venezuela \\ jovalle@usb.ve
}

Received 3 October 2015

Revised 23 October 2015

Published 18 March 2016

\begin{abstract}
By using the extension of the Minimal Geometric Deformation approach, recently developed to investigate the exterior spacetime of a self-gravitating system in the Braneworld, we identified a master solution for the deformation undergone by the radial metric component when time deformations are produced by bulk gravitons. A specific form for the temporal deformation is used to generate a new exterior solution with a tidal charge $Q$. The main feature of this solution is the presence of higher-order terms in the tidal charge, thus generalizing the well known tidally charged solution. The horizon of the black hole lies inside the Schwarzschild radius, $h<r_{s}=2 \mathcal{M}$, indicating that extra-dimensional effects weaken the gravitational field.
\end{abstract}

Keywords: Modified gravity; braneworld; black holes.

PACS numbers: 11.25.-w, 04.50.-h, 04.50.Gh

\section{Introduction}

General Relativity (GR), in its hundred years of existence, has proved to be a successful and well tested theory for gravitation. Along with the Standard Model (SM) of particle physics, both theories represent the fundamental structure of modern physics. However, it is fair to say that GR presents serious problems in the proper description of gravity at both, very small scales and beyond the Solar System scales, namely, its quantum formulation and the dark matter problem. Besides needing dark energy to explain the accelerating universe. This is the main motivation for the

This is an Open Access article published by World Scientific Publishing Company. It is distributed under the terms of the Creative Commons Attribution 4.0 (CC-BY) License. Further distribution of this work is permitted, provided the original work is properly cited. 
search for new theories beyond GR which could explain these fundamental issues. Among them we may mention a large number of extra-dimensional theories and high curvature gravity theories, most of them inspired or a direct consequence of Superstring theory. We also have Galileon theories, $f(R)$ gravity theories, Scalartensor theories, Massive Gravity, New Massive Gravity, Topologically Massive Gravity, Chern-Simons theories, Higher Spin Gravity theories, Horava-Lifshitz Gravity, etc. (see for instance Refs. 1-9). Despite this great effort, the fundamental issues associated with the gravitational interaction remain unresolved.

Among extra-dimensional theories we have the Randall Sundrum Braneworld $(\mathrm{BW})^{10,11}$ which not only explain the hierarchy of fundamental interactions ${ }^{12,13}$ but also is formulated in a non-trivial bulk, which makes it more attractive to explore gravity at high energies as well as generalizations of four-dimensional GR. Because of this, its study and impact on GR is fully justified (see, for instance, Refs. 14-39). In this context, by considering the impact of the BW on self-gravitating system, the Minimal Geometric Deformation (MGD) was developed. ${ }^{40}$ This approach has proven to be useful, among other things, to derive exact and physically acceptable solutions for spherically symmetric and non-uniform stellar distributions ${ }^{41-44}$ as well, to express the tidal charge in the metric found in Ref. 45 in terms of the Arnowitt-Deser-Misner (ADM) mass, ${ }^{46}$ to study microscopic black holes, ${ }^{47}$ to clarify the role of exterior Weyl stresses acting on compact stellar distributions, ${ }^{48,49}$ as well as to extend the concept of variable tension introduced in Ref. 50 by analyzing the black strings solution into the extra dimension, ${ }^{51}$ to prove, contrary to previously established, the existence of Schwarzschild exterior for a spherically symmetric BW self-gravitanting system made of regular matter, ${ }^{52}$ and to derive bounds on extradimensional parameters from the observational results of the classical tests of GR in the Solar system. ${ }^{53}$

In a recent work, ${ }^{54}$ by studying the exterior spacetime $r>R$ associated to a BW self-gravitating system of radius $R$ (where the GR vacuum $r>R$ is filled with a Weyl fluid of extra-dimensional origin), the MGD approach was successfully extended to the most general case for spherically symmetric distributions, namely, when both gravitational potential $g_{t t}$ and $g_{r r}$ are deformed by bulk gravitons. It was shown than the deformation undergone by the temporal metric component produces part of the deformation undergone by the radial metric component. In the present work, by using the extension of the MGD approach and a time deformation parameter, a master solution for the radial geometric deformation associated to the time deformation is identified. Hence by choosing a specific form of the time deformation, a new black hole solution is identified, showing that extra-dimensional effects weaken the gravitational field.

In the next section, we shall present the standard static BW equations for the exterior $r>R$ of a spherically symmetric self-gravitating system, which, contrary to GR, is filled with a Weyl fluid of extra-dimensional origen. In Sec. 2 a brief summary of the generalization of the MGD approach is presented. In Sec. 3 we shall identify a master solution for the radial metric component associated to the time 
deformation, and a new black hole solution is described in detail in Sec. 4. Finally we summarize our work in Sec. 5 .

\section{Field Equations}

In the generalized Randall-Sundrum scenario, gravity acts in the five dimensional bulk $(3+1+1)$ and modifies the gravitational dynamics in the observable fourdimensional world. In the vacuum $T_{\mu \nu}=0$ the effective four-dimensional Einstein equations are written $\operatorname{as}^{\mathrm{a}}$

$$
G_{\mu \nu}=-k^{2} T_{\mu \nu}^{\mathrm{eff}}-\Lambda g_{\mu \nu}
$$

where the effective energy-momentum tensor

$$
T_{\mu \nu}^{\mathrm{eff}}=\frac{1}{8 \pi} \mathcal{E}_{\mu \nu}+\frac{4}{\sigma} \mathcal{F}_{\mu \nu}
$$

encodes all fields with an extra-dimensional origen. Here $\sigma$ is the brane tension and

$$
k^{2} \mathcal{E}_{\mu \nu}=\frac{6}{\sigma}\left[\mathcal{U}\left(u_{\mu} u_{\nu}+\frac{1}{3} h_{\mu \nu}\right)+\mathcal{P}_{\mu \nu}\right],
$$

where $\mathcal{U}$ is the bulk Weyl scalar, $\mathcal{P}_{\mu \nu}$ the stress tensor and $\mathcal{F}_{\mu \nu}$ contains contributions from all non-standard model fields possibly living in the bulk. For simplicity, we shall assume $\mathcal{F}_{\mu \nu}=0$ and $\Lambda=0$ throughout the paper.

In this extra-dimensional context, the exterior $r>R$ of a spherically symmetric self-gravitating system of radius $R$ will be filled with a Weyl fluid of effective density $\mathcal{U}$ and anisotropy $\mathcal{P}_{\mu \nu}$. This fluid has interesting phenomenological consequences, and indeed may be used to explain some issues associated with the gravitational interaction beyond the Solar System scales. ${ }^{55-57}$

By using the Schwarzschild-like coordinates of the metric

$$
d s^{2}=e^{\nu(r)} d t^{2}-e^{\lambda(r)} d r^{2}-r^{2}\left(d \theta^{2}+\sin ^{2} \theta d \phi^{2}\right),
$$

the field equations (1) are written as

$$
\begin{aligned}
& k^{2}\left(\frac{1}{\sigma} \frac{6}{k^{4}} \mathcal{U}\right)=\frac{1}{r^{2}}-e^{-\lambda}\left(\frac{1}{r^{2}}-\frac{\lambda^{\prime}}{r}\right), \\
& k^{2}\left(\frac{1}{\sigma} \frac{2}{k^{4}} \mathcal{U}+\frac{4}{k^{4}} \frac{\mathcal{P}}{\sigma}\right)=-\frac{1}{r^{2}}+e^{-\lambda}\left(\frac{1}{r^{2}}+\frac{\nu^{\prime}}{r}\right), \\
& k^{2}\left(\frac{1}{\sigma} \frac{2}{k^{4}} \mathcal{U}-\frac{2}{k^{4}} \frac{\mathcal{P}}{\sigma}\right)=\frac{1}{4} e^{-\lambda}\left[2 \nu^{\prime \prime}+\nu^{\prime 2}-\lambda^{\prime} \nu^{\prime}+2 \frac{\nu^{\prime}-\lambda^{\prime}}{r}\right],
\end{aligned}
$$

\footnotetext{
${ }^{\text {a }}$ We use $k^{2}=8 \pi G$, where $G$ is the 4 -dimensional Newton constant, and $\Lambda$ is the 4-dimensional cosmological constant.
} 
with primes denoting derivatives with respect to $r$. We also have the conservation equation

$$
\nabla^{\mu} \mathcal{E}_{\mu \nu}=0
$$

which holds when field equations (5)-(7) are satisfied, and the null-fluid condition

$$
\begin{aligned}
R_{\mu}^{\mu}= & e^{-\lambda}\left(\nu^{\prime \prime}+\frac{\nu^{\prime 2}}{2}+2 \frac{\nu^{\prime}}{r}+\frac{2}{r^{2}}\right) \\
& -\lambda^{\prime} e^{-\lambda}\left(\frac{\nu^{\prime}}{2}+\frac{2}{r}\right)-\frac{2}{r^{2}}=0
\end{aligned}
$$

which is the same than the vacuum condition in GR. In this case this expression comes from the condition $\mathcal{E}_{\mu}{ }^{\mu}=0$ for $r>R$ and it is nothing but a linear combination of field equations (5)-(7). From these expressions we identify the density $\mathcal{U}$, effective radial pressure $\tilde{p}_{r}$ and effective tangential pressure $\tilde{p}_{t}$. The effective pressures are given by

$$
\begin{aligned}
& \tilde{p}_{r}=\left(\frac{\mathcal{U}}{3}+\frac{2 \mathcal{P}}{3}\right), \\
& \tilde{p}_{t}=\left(\frac{\mathcal{U}}{3}-\frac{\mathcal{P}}{3}\right),
\end{aligned}
$$

clearly illustrating the anisotropy of the exterior $r>R$, that is

$$
\Pi \equiv \tilde{p}_{r}-\tilde{p}_{t}=\mathcal{P} .
$$

We shall see that the fields $\mathcal{U}$ and $\mathcal{P}$ involves extra-dimensional effects with a big impact on stellar systems. Next a summary of the generalization of the MGD approach is presented.

\section{The Extension of the Minimal Geometric Deformation}

The components of the Weyl fluid $\mathcal{U}(r)$ and $\mathcal{P}(r)$ filling the exterior $r>R$ and the geometric functions $\nu(r)$ and $\lambda(r)$ must satisfy the field equations (5)-(7). Hence it is necessary to provide a condition to close the system. Before imposing any constraint, let us start by using the null-fluid condition in Eq. (9) along with the extended MGD, which considers the deformation undergone by the Schwarzschild solution as

$$
e^{-\lambda(r)}=1-\frac{2 M}{r}+f(r)
$$

and

$$
\nu(r)=\nu_{s}+h(r),
$$

where $\nu_{s}$ is given by the Schwarzschild expression

$$
e^{\nu_{s}}=1-\frac{2 M}{r}
$$


with $f(r)$ the geometric deformation of the radial metric component and $h(r)$ the time deformation. By using the expressions in Eq. (13) and Eq. (14) in the null-fluid condition given in Eq. (9), we obtain the following condition for the exterior $r>R$,

$$
\left(\frac{\nu^{\prime}}{2}+\frac{2}{r}\right) f^{\prime}+\left(\nu^{\prime \prime}+\frac{\nu^{\prime 2}}{2}+\frac{2 \nu^{\prime}}{r}+\frac{2}{r^{2}}\right) f+F(h)=0,
$$

whose formal solution is given by

$$
f(r)=e^{-I\left(r, r_{0}\right)}\left(\beta-\int_{R}^{r} \frac{e^{I(x, R)} F(h)}{\frac{\nu^{\prime}}{2}+\frac{2}{x}} d x\right),
$$

where $\beta$ is an integration constant and the exponent $I=I\left(r, r_{0}\right)$ is given by

$$
I\left(r, r_{0}\right) \equiv \int_{r_{0}}^{r} \frac{\left(\nu^{\prime \prime}+\frac{\nu^{\prime 2}}{2}+\frac{2 \nu^{\prime}}{x}+\frac{2}{x^{2}}\right)}{\left(\frac{\nu^{\prime}}{2}+\frac{2}{x}\right)} d x
$$

where $r_{0}$ is a convenient referential value and with the $F(h)$ function

$$
F(h)=\mu^{\prime} \frac{h^{\prime}}{2}+\mu\left(h^{\prime \prime}+\nu_{s}^{\prime} h^{\prime}+\frac{h^{\prime 2}}{2}+2 \frac{h^{\prime}}{r}\right) .
$$

The exterior deformed radial metric component is finally expressed as

$$
\begin{aligned}
e^{-\lambda(r)}=1 & -\frac{2 M}{r} \\
& +\underbrace{e^{-I(r, R)}\left(\beta-\int_{R}^{r} \frac{e^{I(x, R)} F(h)}{\frac{\nu^{\prime}}{2}+\frac{2}{x}} d x\right)}_{\text {Geometric deformation }} .
\end{aligned}
$$

Finally, the components of the Weyl fluid $\mathcal{U}$ and $\mathcal{P}$ are expressed in terms of the radial deformation $f(r)$ and time deformation $h(r)$ as

$$
\begin{gathered}
\mathcal{U}=-\frac{f}{r^{2}}-\frac{f^{\prime}}{r}, \\
2 \mathcal{P}=\frac{4 f}{r^{2}}+\frac{1}{r}\left[f^{\prime}+3 f \nu_{s}^{\prime}+3 e^{\nu_{s}} h^{\prime}+3 f h^{\prime}\right],
\end{gathered}
$$

where the expressions in Eqs. (14) and (20) have been used in the field equations (5) and (6). Therefore, according to Eq. (20), given a time deformation $h=h(r)$ it will induce part of the radial deformation $f=f(r)$ undergone by the radial metric component. It is worth noting that a vanishing time deformation $h=0$ will produce $F=0$, in consequence the radial geometric deformation will be minimal. For the Schwarzschild geometry, this procedure yields the deformed exterior solution previously studied in Ref. 54 (we note a constant $h$ also produces $F=0$, and corresponds to a time transformation $\left.d T=e^{h / 2} d t\right)$. 


\section{A New Solution}

It would be interesting to consider vacuum solutions by using the deformation parameter $k$ introduced in Ref. 54, namely

$$
e^{\nu}=\left(1-\frac{2 M}{r}\right)^{1+k}
$$

this correspond to a temporal deformation given by

$$
h(r)=k \ln \left(1-\frac{2 M}{r}\right),
$$

where $M$ is a free parameter related with the ADM mass $\mathcal{M}$ of the self-gravitating system. This temporal deformation $h(r)$ induces a deformation in the radial metric component, as clearly is shown in the expression (20), in consequence we will have a modification of the Schwarzschild solution in both gravitational potentials.

Now when Eq. (23) is used in Eq. (20) the deformed radial metric component is given in an exact form by

$$
\begin{aligned}
e^{-\lambda(r)}= & -2 M / r \\
& +\frac{1}{r^{k}}(1-2 M / r)^{(1-k)}[(k-3) M+2 r]^{\frac{3-5 k}{k-3}}\left(\frac{-2 M}{r-2 M}\right)^{k}\left(1+\frac{2 r}{(k-3) M}\right)^{\frac{4 k}{k-3}} \\
& \times\left[\beta r^{\frac{k(k+1)}{k-3}}\left(1-\frac{r}{2 M}\right)^{k}\left(1+\frac{2 r}{(k-3) M}\right)^{\frac{4 k}{k-3}}\right. \\
& \left.-(k-3) M r^{k}(1-2 M / r)[(k-3) M+2 r]^{\frac{4 k}{k-3}} F(r, k)\right],
\end{aligned}
$$

where $F(r, k)$ is the Appell hypergeometric function of two variables, given by

$$
F(r, k)=\operatorname{Apell} F 1\left[a, b_{1}, b_{2}, c ; x, y\right],
$$

where

$$
\begin{aligned}
& a=\frac{k(k+1)}{3-k} ; b_{1}=1-k ; b_{2}=\frac{4 k}{3-k} ; c=\frac{3+k^{2}}{3-k} ; \\
& x=\frac{r}{2 M} ; y=\frac{2 r}{(3-k) M} .
\end{aligned}
$$

The expression in Eq. (25) represents a kind of master equation associated with the time deformation in Eq. (24), in consequence a family of exact configurations may be generated by using the "deformation parameter" $k$ shown in the expression (23). The simplest one is the minimal geometric deformation associated to the Schwarzschild solution, which correspond to $k=0$, namely, no time deformation, yielding to

$$
e^{-\lambda}=\left(1-\frac{2 M}{r}\right)\left(1+\frac{\beta}{1-\frac{3 M}{2 r}} \frac{\ell_{0}}{r}\right),
$$


with

$$
\ell_{0} \equiv R \frac{\left(1-\frac{3 M}{2 R}\right)}{\left(1-\frac{2 M}{R}\right)} .
$$

Of course when $\beta=0$ we regain the well known Schwarzschild solution. A more interesting case, still not considered, is the one where $k=4$, which represents a solution with high order deformation in terms of a tidal charge $Q$

$$
e^{\nu}=1-\frac{2 \mathcal{M}}{r}+\frac{Q}{r^{2}}-\frac{2}{5} \frac{\mathcal{M} Q}{r^{3}}+\frac{Q^{2}}{20 r^{4}}-\frac{\mathcal{M} Q^{2}}{250 r^{5}},
$$

where $\mathcal{M}=5 M$ and $Q=40 M^{2}$. The tidal charge $Q$ is nothing but extradimensional effects producing deviation from the Schwarzschild's solution. Similarly the radial metric component is expressed in an exact form, too large to display here. This solution displays a zero of $g_{r r}^{-1}$, namely a horizon $r=h$, and a surface $r=r_{c}$ where $g_{r r}^{-1}$ diverges (and $g_{t t}=0$ ), all shown in Fig. 1 . These surfaces separate the space-time in three regions, namely

- $0<r<r_{c}$,

- $r_{c}<r<h$,

- $r>h$.

We want to emphasize that an exterior observer at $r>h$ will never see the singularity at $r=r_{c}$, as it is hidden behind the horizon $r=h$. Finally, since the horizon lies inside the Schwarzschild radius, $h<r_{s}=2 \mathcal{M}$, this solution clearly indicates that extra-dimensional effects weaken the gravitational field. The solution also describes the exterior of a stellar structure with radius $R>h$.

Both Weyl functions $\mathcal{U}$ and $\mathcal{P}$ are shown in Fig. 2 and Fig. 3 respectively and compared with the case $k=2$. While the scalar $\mathcal{U}(r)$ is always positive, the Weyl

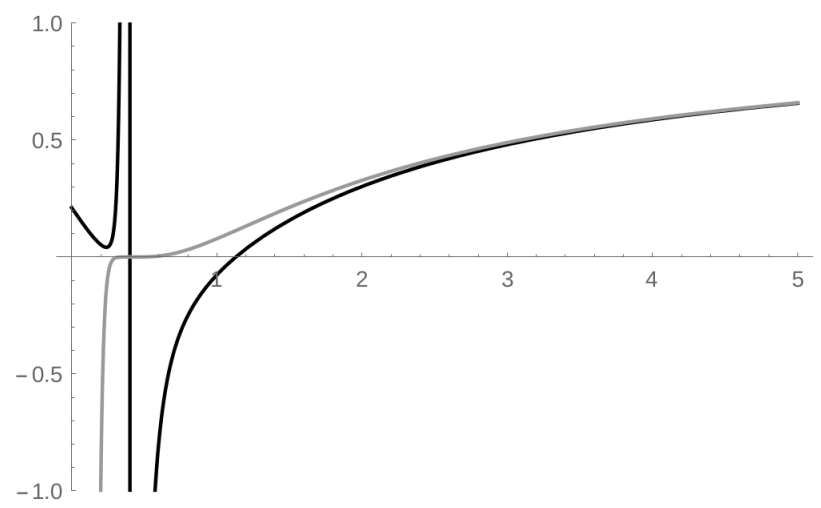

Fig. 1. Behaviour of $g_{t t}(r)$ (Gray) and $g_{r r}^{-1}(r)$ (Black) for $k=4$. We see a zero and a singular point for $g_{r r}^{-1}$. The singularity at $r_{c}=2 / 5$ is hidden behind the horizon $h \simeq 1.131$. It can be seen that the black hole horizon is shifted inside the Schwarzschild radius $\left(r_{s}=2 \mathcal{M}\right)$ by extra-dimensional effects. The ADM mass is $\mathcal{M}=1$. 


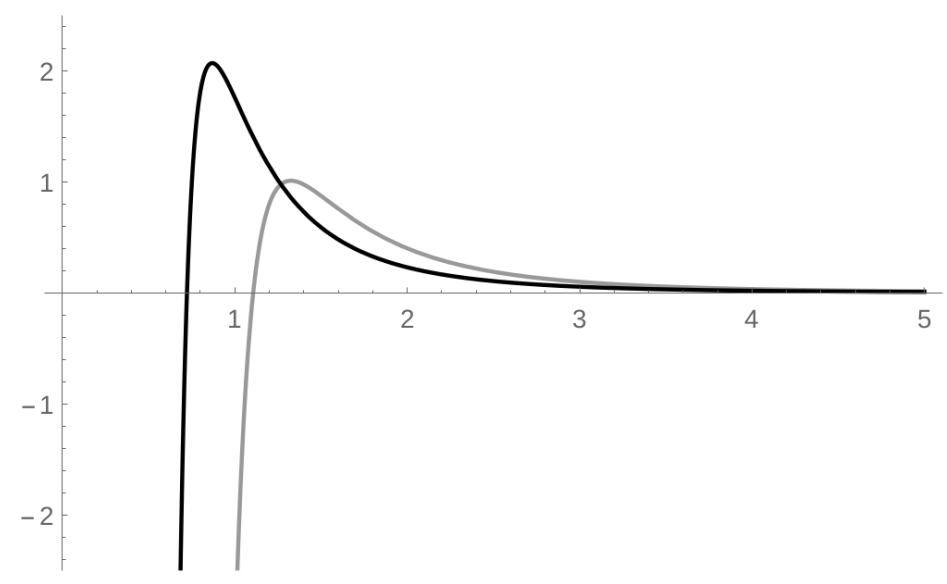

Fig. 2. Comparison of the effective density $\mathcal{U}(r)$ for the case $k=2$ (gray) and $k=4$ (black). In both cases the scalar function $\mathcal{U}$ increasing as we approach to the distribution until reach a maximum value. This maximum is slightly beyond the horizon for $k=2$ and inside it for $k=4$. In both cases the function diverges as we approach to their respective singularities $r_{c}$. The black hole mass is setting as $\mathcal{M}=1$.

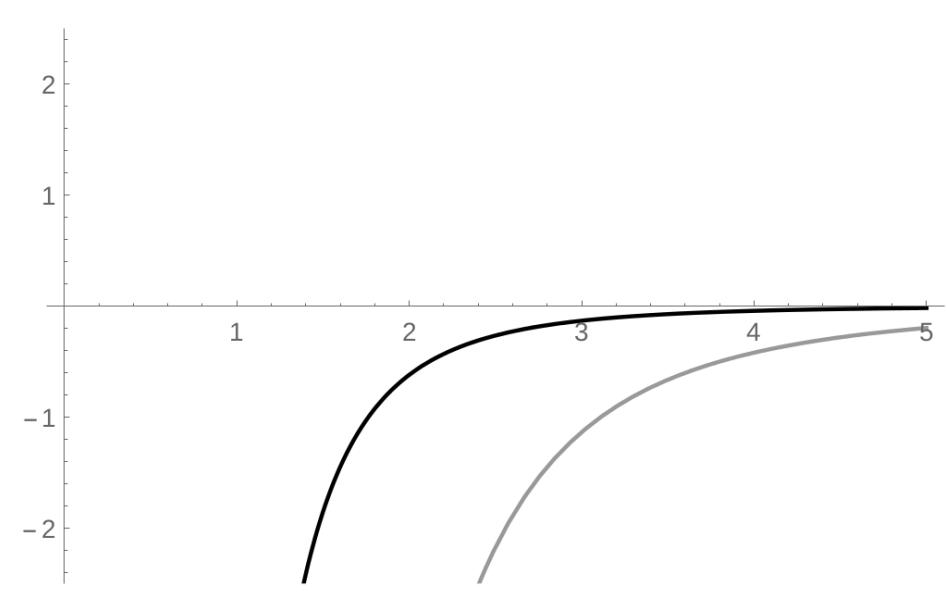

Fig. 3. Comparison of the anisotropy $\mathcal{P}(r)$ for the case $k=2$ (gray) and $k=4$ (black). In both cases $\mathcal{P}$ is incremented negatively and diverges as we approach to their respective singularities $r_{c}$. The black hole mass is setting as $\mathcal{M}=1$.

functions $\mathcal{P}(r)$ is negative. This indicates a negative radial deformation and also a positive temporal deformation, according to Eqs. (21) and (3), and diverges at the singular surface $r=r_{c}$. Both functions represent the Weyl fluid around the self-gravitating system as consequences of extra-dimensional effects. It is interesting to note that in this new case with $k=4$ the Weyl fluid is weaker than the studied in Ref. 54 with $k=2$. 


\section{Conclusions}

In the context of the BW, and by using the extension of the MGD approach, where both gravitational potentials are deformed by bulk gravitons, the exterior spacetime $r>R$ associated to a BW self-gravitating system of radius $R$ was studied, and the consequences of extra-dimensional effects on the Schwarzschild solution were investigated. By using the specific time deformation $h(r)$ in Eq. (24), a master equation showing the deformation undergone by the radial metric component was identified in Eq. (25). This master solution depends on three free parameters, the GR mass $M$ (ADM mass without bulk effects), the time parameter $k$, and an integration constant $\beta$ which is fixed demanding the Schwarzschild limit $g_{r r}^{-1} \sim 1-2 \mathcal{M} / r$, where the ADM mass $\mathcal{M}$ and the GM mass $M$ are related by $\mathcal{M}=(1+k) M$.

Taking a specific value for the time parameter, namely $k=4$, a new exact exterior solution for a spherically symmetric self-gravitating systems was identified. The main feature of this solution is the presence of higher-order terms in the tidal charge $Q$, thus generalizing the well known tidally charged black hole solution found in Ref. 45. This new solution, which has a singularity $r_{c}$ hidden behind the horizon $h$, represents a black hole whose horizon lies inside the Schwarzschild radius, $h<$ $r_{s}=2 \mathcal{M}$, indicating that extra-dimensional effects weaken the gravitational field. The scalar function $\mathcal{U}$ increasing as we approach to the distribution until reach a maximum value inside the horizon $h$, then it diverges as we approach to the singularity $r_{c}$. The opposite behavior is seen for $\mathcal{P}$, which is always negative. Both functions tend to disappear rapidly as we move away from the stellar distribution, showing thus a "Weyl atmosphere" surrounding the self-gravitating system. The new solution was compared with the previous one obtained in Ref. 54. Despite the fact that in this new solution the time deformation is greater than the one in Ref. 54, the Weyl fluid surrounding the self-gravitating system is weaker. This shows that the simplest way to introduce a deformation on the temporal metric component, namely the expression shown in Eq. (23), has non trivial consequences. In this respect, it would be interesting to use the classical tests of General Relativity in the Solar system, as done in Ref. 53, to derive bounds on the time parameter $k$, as well as to investigate the extension of this solution in the complete five-dimensional bulk to clarify the role of $k$. Finally, despite the fact that in the braneworld it is not clear whether the process of gravitational collapse leaves a signature in the black hole end-state or not, ${ }^{58-60}$ the study of the "no hair theorem" by using these higher-order tidal charge solutions represents an attractive scenario.

\section{Acknowledgments}

The author thanks the Abdus Salam International Center for Theoretical Physics, ICTP, for hospitality and financial support.

\section{References}

1. E. Bergshoeff, O. Hohm and P. K. Townsend, Phys. Rev. Lett. 102, 201301 (2009). 
2. C. de Rham, Living Rev. Relativity 17, 7 (2014).

3. N. Chow and J. Khoury, Phys. Rev. D 80, 024037 (2009).

4. A. De Felice and S. Tsujikawa, Living Rev. Rel. 13, 3 (2010).

5. T. P. Sotiriou and V. Faraoni, Rev. Mod. Phys. 82, 451 (2010).

6. S. Capozziello and M. De Laurentis, Phys. Rept. 509, 167 (2011).

7. S. Capozziello, V. F. Cardone and A. Troisi, Phys. Rev. D 71, 043503 (2005).

8. T. Clifton, P. G. Ferreira, A. Padilla and C. Skordis, Phys. Rept. 513, 1 (2012).

9. P. Horava, Phys. Rev. D 79, 084008 (2009).

10. L. Randall and R. Sundrum, Phys. Rev. Lett. 83, 3370 (1999).

11. L. Randall and R. Sundrum, Phys. Rev. Lett. 83, 4690 (1999).

12. N. Arkani-Hamed, S. Dimopoulos and G. Dvali, Phys. Lett. B 429, 263 (1998).

13. I. Antoniadis, N. Arkani-Hamed, S. Dimopoulos and G. Dvali, Phys. Lett. B 436, 257 (1998).

14. T. Shiromizu, K. Maeda and M. Sasaki, Phys. Rev. D 62, 024012 (2000).

15. C. Germani and R. Maartens, Phys. Rev. D 64, 124010 (2001).

16. R. da Rocha and J. M. Hoff da Silva, Phys. Rev. D 85, 046009 (2002).

17. R. Casadio, A. Fabbri and L. Mazzacurati, Phys. Rev. D 65, 084040 (2002).

18. P. Kanti and K. Tamvakis, Phys. Rev. D 65, 084010 (2002).

19. G. Kofinas, E. Papantonopoulos and V. Zamarias, Phys. Rev. D 66, 104028 (2002).

20. G. Kofinas, E. Papantonopoulos and I. Pappa, Phys. Rev. D 66, 104014 (2002).

21. L. A. Gergely, Phys. Rev. D 68, 124011 (2003).

22. L. A. Gergely, Phys. Rev. D 78, 084006 (2003).

23. R. Casadio and L. Mazzacurati, Mod. Phys. Lett. A 18, 651 (2003).

24. P. Kanti, I. Olasagasti and K. Tamvakis, Phys. Rev. D 68, 124001 (2003).

25. M. Visser and D. L. Wiltshire, Phys. Rev. D 67, 104004 (2003).

26. A. S. Majumdar and N. Mukherjee, Int. J. Mod. Phys. D 14, 1095 (2005).

27. S. Creek, R. Gregory, P. Kanti and B. Mistry, Class. Quantum Grav. 23, 6633 (2006).

28. L. A. Gergely, J. Cosmol. Astropart. Phys. 02, 027 (2007).

29. R. Casadio and O. Micu, Phys. Rev. D 81, 104024 (2010).

30. R. Maartens and K. Koyama, Living Rev. Rel. 13, 5 (2010).

31. D. C. Dai and D. Stojkovic, Phys. Lett. B 704, 354 (2011).

32. R. da Rocha and J.M. Hoff da Silva, Phys. Rev. D 85, 046009 (2012).

33. S. Abdolrahimi, C. Catton, D. N. Page and S. Yaghoobpour-Tari, Phys. Lett. B 720, 405 (2013).

34. D. Bazeia, J. M. Hoff da Silva and R. da Rocha, Phys. Rev. D 90, 047902 (2014).

35. T. Harko and M. J. Lake, Phys. Rev. D 89, 064038 (2014).

36. L. B. Castro, M. D. Alloy and D. P. Menezes, JCAP 1408, 047 (2014).

37. T. Harko and M. J. Lake, Phys. Rev. D 89, 064038 (2014).

38. S. Chakraborty and S. SenGupta, Eur. Phys. J. C 75, 11 (2015).

39. F. X. Linares, M. A. Garcia-Aspeitia and L. A. Urea-Lopez, Phys. Rev. D 92, 024037 (2015).

40. J. Ovalle, Braneworld stars: anisotropy minimally projected onto the brane, in Gravitation and Astrophysics (ICGA9), ed. J. Luo (World Scientific, Singapore, 2010), p. 173.

41. J. Ovalle, Mod. Phys. Lett. A 23, 3247 (2008).

42. J. Ovalle, Int. J. Mod. Phys. D 18, 837 (2009).

43. J. Ovalle, Mod. Phys. Lett. A 25, 3323 (2010).

44. J. Ovalle, Effects of density gradients on braneworld stars, in Proceedings of the Twelfth Marcel Grossmann Meeting on General Relativity, eds. T. Damour, R. T. Jantzen and R. Ruffini (World Scientific, Singapore, 2012), p. 2243. 
45. N. Dadhich, R. Maartens, P. Papadopoulos and V. Rezania, Phys. Lett. B 487, 1 (2000).

46. R. Casadio and J. Ovalle, Phys. Lett. B, 715, 251 (2012).

47. R. Casadio and J. Ovalle, Gen. Relat. Grav. 46, 1669 (2014).

48. J. Ovalle and F. Linares, Phys. Rev. D 88, 104026 (2013).

49. J. Ovalle, F. Linares, A. Pasqua and A. Sotomayor, Class. Quantum Grav. 30, 175019 (2013).

50. L. A. Gergely, Phys. Rev. D 78, 084006 (2008).

51. R. Casadio, J. Ovalle and R. da Rocha, Class. Quantum Grav. 30, 175019 (2014).

52. J. Ovalle, L. A. Gergely and R. Casadio, Class. Quantum Grav. 32, 045015 (2015).

53. R. Casadio, J. Ovalle and R. da Rocha, Europhys. Lett. 110, 40003 (2015).

54. R. Casadio, J. Ovalle and R. da Rocha, Class. Quantum Grav. 32, 215020 (2015).

55. M. K. Mak and T. Harko, Phys. Rev. D 70, 024010 (2004).

56. C. G. Boehmer and T. Harko, Class. Quantum. Grav. 24, 3191 (2007).

57. L. A. Gergely, T. Harko, M. Dwornik, G. Kupi and Z. Keresztes, Mon. Not. R. Astron. Soc. 415, 3275 (2011).

58. C. Germani, M. Bruni and R. Maartens, Phys. Rev. Lett. 87, 1 (2001).

59. R. Emparan, A. Fabbri and N. Kaloper, J. High Energy Phys. 2002, 043 (2002).

60. T. Wiseman, Phys. Rev. D 65124007 (2002). 